\title{
Philosophiques
}

\section{Roberto Miguelez, Science, valeurs et rationalité, Collection des sciences sociales, no 8, Ottawa, Éditions de l'Université d'Ottawa, 1984, 95 p.}

\section{J. N. Kaufmann}

Volume 12, numéro 1, printemps 1985

URI : https://id.erudit.org/iderudit/203284ar

DOI : https://doi.org/10.7202/203284ar

Aller au sommaire du numéro

Éditeur(s)

Société de philosophie du Québec

\section{ISSN}

0316-2923 (imprimé)

1492-1391 (numérique)

Découvrir la revue

Citer ce compte rendu

Kaufmann, J. N. (1985). Compte rendu de [Roberto Miguelez, Science, valeurs et rationalité, Collection des sciences sociales, no 8, Ottawa, Éditions de

l’Université d'Ottawa, 1984, 95 p.] Philosophiques, 12(1), 225-229.

https://doi.org/10.7202/203284ar d'utilisation que vous pouvez consulter en ligne.

https://apropos.erudit.org/fr/usagers/politique-dutilisation/ 
ROBERTO MIGUELEZ, Science, valeurs et rationalité, Collection des sciences sociales, no 8, Ottawa, Éditions de l'Université d'Ottawa, 1984, 95 p.

\author{
par J.N. Kaufmann
}

Depuis un siècle et demi, la question de la neutralité axiologique de la science surgit à intervalles réguliers et reçoit des réponses variables, de J.S. Mill, S. Jevons, M. Weber, G. Myrdal à Jürgen Habermas. Qu'elle se pose à l'époque contemporaine avec une acuité de plus en plus nette, comme en témoigne le texte de $\mathrm{R}$. Miguelez, ne signifie pas qu'elle ait été absente à d'autres époques. La science de Galilée ne fut pas perçue comme ce tribunal indépendant qui ne jugerait qu'à partir des faits du monde tels qu'ils se révéleraient eux-mêmes. Elle fut comprise comme menace portant atteinte à un système de valeurs établies. Cela signifie qu'elle fut perçue comme porteuse de valeurs. Mais en quel sens au juste ? C'est cela que R. Miguelez essaie de préciser dans son livre. En s'inspirant très nettement des thèses weberiennes sur la neutralité axiologique et la rationalité de l'action, pour en prendre cependant la distance sur des points précis, en prolongeant la critique que Habermas a faite de la rationalité technique, et en se servant avec beaucoup d'à propos des résultats de l'épistémologie scientifique que préconise l'empirisme logique, $R$. Miguelez formule quatre thèses (en introduction, p. 10) qu'il soutient tout au long de son texte par une argumentation très serrée. Les voici. 1) Les paradigmes scientifiques délimitent des espaces axiologiques. Ils rendent ainsi possibles certaines valeurs et en excluent d'autres. 2) Les découvertes scientifiques qui s'expriment dans des énoncés nomologiques induisent des valeurs en ouvrant l'horizon à des actions possibles et en rendant caduques d'autres valeurs. 3) La rationalité scientifique technique prescrit un modèle de comportement et se présente comme valeur centrale dans les représentations idéologiques dominantes. 4) La rationalité technique doit être pensée dans son rapport à la manière dont l'idéologie technocratique définit l'« acteur social " comme « sujet ».

Distinguant soigneusement la science comme discours, comme activités et processus réglés, et comme application de ses résultats dans la production technologique non créative, séparant les questions de logique et d'épistémologie de celles qui relèvent de préférences et de décisions pratiques, $R$. Miguelez examine les deux premières thèses (dans la première partie du livre) et indique à l'intérieur de quelles limites et sous quelles formes la thèse, très générale, de la neutralité axiologique de la science pourrait être défendable. (Les différentes possibilités se trouvent bien résumées dans un tableau, p. 21). La science en tant qu'entreprise cognitive poursuit un objectif : dire vrai. La vérité est déclarée valeur décisive que présuppose la science et, selon Max Weber, cette valeur peut être considérée comme indépendante de toutes les autres. Mais cela n'est guère précis aussi longtemps que l'on n'indique pas laquelle des théories de la vérité (théorie correspondantiste de la vérité, théorie de l'évidence, théorie " consensuelle » de la vérité, théorie pragmatique, c'est-à-dire est vrai ce qui est efficace . . .) les scientifiques incorporent dans leur entreprise. "Chercher la vérité » est bien trop vague pour être considéré 
comme un objectif véritable. (Est-ce la raison pour laquelle on parle alors de valeur plutôt que d'objectif ?). Même si l'on apportait des précisions en identifiant, par exemple, la construction de systèmes (de propositions acceptées comme vraies) explicatifs de plus en plus englobants et généraux, ou l'approximation de plus en plus parfaite entre les data prédits et les data observés, ou encore l'augmentation de la capacité d'une théorie de prédire des effets nouveaux, comme buts de la science, il n'est pas évident que les scientifiques s'accorderaient à ce sujet. Mais une formulation précise des objectifs (cognitifs) serait requise pour apprécier, comme adéquates ou inadéquates (bref, pour appliquer les standards de rationalité scientifique à l'entreprise scientifique elle-même), les normes méthodologiques et instrumentales régissant l'activité scientifique et pour définir plus clairement la notion d' « utilité " d'une hypothèse et les critères de crédibilité ou d'acceptation de celle-ci. Cela permettrait de distinguer l'utilité épistémique d'une hypothèse (par rapport à des objectifs purement cognitifs de la science) de son utilité pratique dépendant des conséquences pratiques découlant d'une hypothèse confirmée). Cela permettrait aussi de reprocher à Weber d'avoir simplifié énormément non seulement ce qui apparaît comme "wissenswert " (en réduisant ce qui est " digne de savoir " à une décision individuelle), mais aussi les aspects ayant trait aux objectifs cognitifs de l'entreprise scientifique, en mettant de la sorte des choses différentes sous le titre de "vérité " pour ensuite déclarer que «la vérité » était l'unique valeur poursuivie par la science.

D'autre part, la thèse neutraliste sous-entend que si les différentes disciplines proposent ou véhiculent des valeurs particulières dans le sens que ces valeurs déterminent le choix des problèmes que les disciplines en question sont appelées à reformuler dans leur cadre théorique respectif à l'aide d'hypothèses spécifiques conduisant à des prolongements techniques qui, eux, sont franchement dépendants des valeurs et des normes prescrivant ou interdisant l'usage de ces technologies, on peut alors remarquer que ces prescriptions de valeur n'ont pas d'intérêts épistémologiques si elles ne déterminent pas la nature des produits cognitifs.

Cependant $R$. Miguelez montre de façon très convaincante, par une analyse formelle de la situation pragmatique comportant une règle d'action ( nomotechnique ») fondée sur un énoncé nomologique, que la science n'impose peut-être pas de valeurs déterminées, mais elle détermine à tout le moins des champs d'actions possibles ainsi que le domaine des valeurs correspondant. R. Miguelez obtient ce résultat en explicitant de façon originale la formulation lapidaire que donne Bunge (1967, II, 11.2) des relations de présupposition entre des énoncés nomologiques (du type implication $\mathrm{p} \rightarrow$ q), des énoncés nomopratiques (du type : "En faisant en sorte que p, q se réalise "), des énoncés normatifs (du type des impératifs conditionnels : « Si tu veux réaliser $q$, alors il faut faire $\mathrm{p}$ ), ainsi que les énoncés de préférences ("A préfere q à toute autre option) et les jugements de valeurs correspondants ( Pour A, q réalise la valeur $Q$ »). Ces relations sont représentées dans la formule : 
«p $\rightarrow \mathrm{q}$ " fund (« q per $\mathrm{p}$ », parce que « q " est voulu par $\mathrm{A}$ ), et ( $\mathrm{q}$ » est voulu par $A$ présuppose $Q$ ).

En supposant que certains états du monde (q) possibles sont souhaitables et désirés comme des fins de l'action, on peut remarquer que la science ne sert pas directement à fixer ces buts, mais qu'elle permet de déterminer les moyens adéquats pour $y$ parvenir en permettant de "dériver " des règles technologiques ; plus exactement les énoncés nomologiques qui sont du ressort de la science sont des conditions nécessaires pour la formulation des règles technologiques. C'est en cela que consiste, selon Max Weber, la Zweckrationalitët que prescrit la science. C'est donc elle qui délimite un champ d'actions possibles et ouvre par là un champ de valeurs possibles (p. 43). Qu'il s'agisse d'une imposition (p. 43) de valeurs est une autre question. Il y a sans doute orientation par la science des actions futures possibles, mais les agents à qui s'adresseraient les règles techniques " découlant " de $\mathrm{p} \rightarrow \mathrm{q}$ pourraient ne pas être satisfaits et refuser d'agir en vertu de ces prescriptions. Ils pourraient vouloir envisager plusieurs options et demander ainsi à la science si $\mathrm{p}^{\prime} \rightarrow \mathrm{q}$ ou $\mathrm{p}^{\prime \prime} \rightarrow \mathrm{q}$ satisfont également les critères d'acceptation, de sorte qu'on puisse choisir parmi plusieurs règles techniques. La tâche de la science est alors d'indiquer à l'intérieur de quelles limites - des limites qui sont toujours repoussées plus loin avec le temps - les choix sont possibles, et au delà desquelles il n'y a plus d'options.

Il apparaît donc clairement que certaines actions plutôt que d'autres sont rendues possibles par la science. Mais il faudrait maintenant montrer comment la science contribue à faire en sorte que des individus ou des groupes concrets réalisent effectivement ces possibilités pour concrétiser de la sorte la ou les valeurs induites par la science. Et cela signifie qu'on puisse expliquer pourquoi certaines valeurs, un champ de valeur ouvert par la science (q), sont adoptées par des agents déterminés. R. Miguelez n'a pas réussi, dans les passages où il aborde ce problème (notamment dans la section 2), à démontrer cette possibilité. Il se contente de proposer une telle explication comme idée régulatrice dont la structure est au fond double : expliquer des actions à partir des intentions, croyances (les idées scientifiques permettant de fonder des règles techniques), préférences et valeurs des agents, et expliquer en même temps la genèse de ces attitudes psychologiques à partir de la situation des agents.

C'est dans la troisième partie que $R$. Miguelez examine le rôle que pourraient jouer les éléments de l'action («but ", " moyen ", croyances concernant leurs rapports, etc.) dans une variété de modèles d'explication de l'action (théorie de l'action de Weber et de Parsons, interactionnisme symbolique de C.H. Mead et Blumer et de la sociologie phénoménologique de Schütz et de l'ethnométhodologie de Garfinkel.), lesquels modèles relèveraient du «paradigme de la définition sociale » (p. 65). Toutes ces formes d'explications se rapprochent tantôt du schéma de l'explication rationnelle de Hempel (1965), tantôt de l'inférence pratique de von Wright (1971). L'intérêt de ces reconstructions très succinctes est de démontrer clairement 
que ces « explications " présupposent et mettent en jeu une idée particulière de rationalité dans l'action, à savoir la Zweckrationalität (« la rationalité par fin " de Weber ou la "rationalité technique " de Habermas). Celle-ci joue dans les arguments explicatifs ou bien un rôle purement analytique ou définitionnel (ce qui n'est pas suffisamment souligné dans le texte), ou alors elle y figure comme concept normatif. C'est en soulignant ce dernier point que R. Miguelez veut établir sa troisième thèse concernant l'investissement axiologique de l'explication "scientifique " des actions et la prise en charge par celle-ci d'un modèle de rationalité de l'action.

Pour se distancier de Max Weber, R. Miguelez oppose à la Zweckrationalität qui est en fait un modèle de rationalité individuelle et qui est à la base de l'idée centrale caractérisant l'économie capitaliste (le meilleur ordre économique s'établira à condition que chacun maximise ses fonctions d'utilité individuelles, choisisse les moyens scientifiquement les plus adéquats en vue de certaines fins, c'est-à-dire que chacun agisse selon un modèle de rationalité individuelle) l'idée d'une rationalité collective, d'une rationalité des structures sociales. Conformément à la critique développée par Habermas, il maintient que la rationalité instrumentale scientificotechnique, promue au cours de l'histoire récente, notamment avec le succès du capitalisme qui s'en réclame, comme la forme supérieure de rationalité, est devenue, au nom d'une notion limitée et illusoire de l'individu comme " sujet ", un instrument de domination technocratique. En effet " la science comme idéologie et l'idée du sujet (individuel) et de la rationalité (instrumentale) ne font alors qu'un seul ensemble de représentations articulées ». (p. 91).

Je signale qu'une telle critique de la rationalité économique qui ne se réclamerait que de la rationalité individuelle se trouve formulée bien avant Habermas par un célèbre représentant de la théorie économique néo-classique (capitaliste). Il s'agit de O. Morgenstern (1928), fondateur de la théorie des jeux. En effet, quand Morgenstern formule les problèmes épistémologiques et paradoxes inhérents à la théorie générale de l'équilibre, il montre que dans des situations d'interaction, comme dans une situation d'échange de marchandises, le problème du choix consiste en une décision où il ne faut pas seulement tenir compte des variables « mortes " qui représentent les contraintes habituelles pour un problème de maximisation, mais aussi de variables " vivantes ", à savoir des décisions des autres agents. Il s'agit alors d'une situation tout à fait nouvelle, c'est-à-dire d'un problème de choix sous incertitude stratégique. Dans ce cas il ne s'agit plus de voir seulement comment des individus, pour agir rationnellement selon le paradigme dominant au sens de la rationalité individuelle, maximiseraient leur fonction d'utilité. C'est un problème de décisions interdépendante dont la solution consiste à savoir comment les actions de chacun peuvent en quelque sorte se compatibiliser et concourir à un résultat global, comme par exemple à un équilibre de marché. Une telle solution relève pour von Neumann et Morgenstern (1944) de la théorie des jeux, qui introduit explicitement une nouvelle conception de la rationalité qui fut partiellement incorporée à la théorie économique capitaliste. Mais cette rationalité n'a aucun sens sur le plan individuel : 
elle n'apparaît qu'au niveau de l'interaction des individus et consiste en la conformité de ceux-ci à la stratégie optimale. Cela constitue un phénomène d'émergence d'une rationalité structurelle ou collective qui ne semble pas aussi étrangère aux " théories économiques capitalistes " que pourraient le croire J. Habermas et R. Miguelez.

Département de philosophie

Université du Québec à Trois-Rivières 